\title{
ANTI-FATIGUE MATS, LOW BACK PAIN, AND ELECTROMYOGRAPHY: AN INTERVENTIONAL STUDY
}

\section{JAVAD AGHAZADEH ${ }^{1}$, MAHMOUD GHADERI ${ }^{2}$, MAHMOOD-REZA AZGHANI ${ }^{3}$, HAMID-REZA KHALKHALI ${ }^{4}$, TEIMOUR ALLAHYARI ${ }^{2}$, and IRAJ MOHEBBI ${ }^{5}$}

${ }^{1}$ Tabriz University of Medical Sciences, Tabriz, Iran

Neurosurgery Department

${ }^{2}$ Urmia University of Medical Sciences, Urmia, Iran

Occupational Health Department

${ }^{3}$ Sahand University, Tabriz, Iran

Biomechanic Department

${ }^{4}$ Urmia University of Medical Sciences, Urmia, Iran

Biostatistics and Epidemiology Department

${ }^{5}$ Urmia University of Medical Sciences, Urmia, Iran

Social Determinants of Health Research Center, Occupational Medicine Center

\begin{abstract}
Objectives: Increasing bilateral gluteus medius co-activation has been identified as one of the most important factors in developing low back pain due to prolonged standing in healthy people. This study aims to investigate the impact of an anti-fatigue mat on the bilateral gluteus medius co-activation pattern and to report the low back pain subjectively in 2 different standing positions on the normal rigid surface and on the anti-fatigue mat. Material and Methods: While carrying out an easy simulated profession, 16 participants who had no low back pain background were requested to stand for $2 \mathrm{~h}$ in each position, with and without using the anti-fatigue floor mat, respectively. At the beginning of standing process and at every 15 min until the time of 120 min lapses, electric activities for the bilateral gluteus medius co-activation and subjective pain level in low back area were collected by the surface electromyogeraphy (EMG) and the visual analogue scale (VAS), respectively in each position. Results: The obtained findings revealed that the anti-fatigue mat significantly decreased subjective pain level in low back area among 15 participants $(p<0.05)$. However, there was objectively no significant difference in the bilateral gluteus medius co-activation pattern among the participants between the position 1 and the position $2(\mathrm{p}>0.05)$. The findings obtained under this study related to the impact of the anti-fatigue mat upon the low back pain based on the increase of $>10 \mathrm{~mm}$ on the VAS threshold, which showed that this intervention had no significant impact upon decreasing the number of patients suffering from the low back pain and also minimizing the bilateral gluteus medius co-activation in both pain developer groups ( $\mathrm{p}>0.05)$. However, $73 \%$ of the participants preferred to apply it. Conclusions: It seems that the anti-fatigue mat may be useful in reducing the low back pain although it objectively didn't significantly change the gluteus medius co-activation pattern related to the low back pain.
\end{abstract}

Key words:

Low back pain, Anti-fatigue mat, Muscle co-activation, EMG

The source of financial support: Urmia University of Medical Sciences. The project titled "The anti-fatigue mats and low back pain: A surface electromyography study of gluteus medius muscles co-activation". The project manager: Prof. Iraj Mohebbi.

Received: April 27, 2014. Accepted: September 19, 2014.

Corresponding author: I. Mohebbi, Occupational Medicine Center, Urmia University of Medical Sciences, Post Box: 5756115111, Urmia, Iran (e-mail: mohebbi_iraj@yahoo.co.uk). 


\section{INTRODUCTION}

Low back pain is one of the most prominent work-related musculoskeletal disorders. Prevalence of this disorder has been estimated to stand at $60-80 \%$, and to occur mostly in 2 nd to 5th decades of life [1]. Epidemiologic studies have indicated that there is a significant relationship between developing the low back pain and prolonged standing [2]. Prolonged standing is common among service workers such as cashiers, manufacturing occupations such as assembly line workers, inspectors and quality control workers as well as nurses and surgeons in health care sections [3-7].

It seems that the low back pain is mainly due to the static posture during prolonged standing and developing and accumulating metabolites in low back muscles [8]. Nevertheless, there are different reasons for the low back pain in most mechanism cases. Therefore, it remains to be proven how to predict the pain effectively [9]. Biomechanical and kinesiological studies have been conducted to identify the predicting factors of low back pain due to prolonged standing among people lacking symptoms of low back pain. These investigators tried to assess various variables such as hip and torso muscles activation, lumbar spine posture, oxygenating in back extensor muscles, torso skin temperature, center of pressure center changes, strength, gluteus medius muscles activation and endurance tolerance, trunk muscles activation, and hamstring muscles tight. It is worth noting that these variables have been investigated in some studies about clinical and psychosocial assessment instruments [6-12].

The effective factors in identifying individuals who are susceptive to low back pain were as the following: trunk flexor/extensor and bilateral gluteus medius muscles coactivation patterns, frontal plane control during clinical assessment and gluteus medius endurance, based on the visual analogue scale (VAS), in the pain developer group as compared to the non-pain developer group, the threshold showed an increase in the trunk flexor/extensor and bilateral gluteus medius muscles co-activation. A significant decrease in frontal plane control and in gluteus medius endurance [8-11] had already been shown. Despite the basis factor as a dominant and reliable pattern in all studies, bilateral gluteus medius muscles co-activation and individuals' subjective report on low back pain are based on the VAS, so that $76-79 \%$ of the individuals can be accordingly divided into 2 pain developer and non-pain developer groups [8-10,13].

Low back pain due to prolonged standing has a considerable impact upon health, sick absence and productivity [3,14]. Accordingly, many countries have broadly studied prolonged standing as an ergonomic problem quantitatively [3].

Multiple ergonomic interventions have been developed in order to reduce low back pain resulting from prolonged standing. These interventions aim to make appropriate workplace for workers and staff. Some of them are: changing a mat under the feet of workers, using shock-absorbing shoe insole, using arch-support orthotics, wearing shockabsorbing shoes and applying foot rail [3-5,14-22].

Among these interventions, the anti-fatigue floor mat is a common ergonomic solution in decreasing complications arising from prolonged standing [3,14]. Mats are mainly applied in industries. In addition, a special mat has been designed for surgery in the health care section to minimize the effects arising from prolonged standing in surgeries [4].

Various mats have been evaluated and compared according to features such as stiffness, thickness, compression, material quality and their relationship with fatigue and discomfort in order to identify an effective mat in reducing complaints arising from prolonged standing. Several studies indicated that a soft mat significantly decreased pain level $[3,14,17,20,23]$. For thickness, Chaffin and Redfern stated that 3.8-5.8-inch mats are the most effective ones [20]. According to Konz et al., the least compressed mats are the best [24]. Also, Rys and Konz came to the conclusion that 
mats having $6 \%$ compression due to body weight are more comfortable than those having 18\% compression [25].

However, Cham and Redfern have revealed that there are factors such as elasticity increase, reduction of energy absorbance, and mat stiffness increase that lead to discomfort and fatigue alleviation after standing for $3 \mathrm{~h}$ in a 4-h standing pattern [17].

Many studies have been conducted to investigate the effectiveness of these interventions in low back and lower limb complications resulting from prolonged standing. The effect of the anti-fatigue floor mat on different subjective and objective variables related to standing has been investigated in these studies. In the studies on the subjective criteria of individuals in different surface positions, although most results showed that the mats had a significant impact upon individuals' understanding of fatigue and discomfort $[3,17,20]$, it is nearly incompatible with the results obtained from studies conducted on the effect of mats on some special body areas [14]. On the other hand, recording local fatigue in paraspinal muscles using the surface electromyography (EMG) was the only objective, achievement of which was assessed in order to determine the effect of mats on the fatigue and discomfort in low back area. In previous studies among biomechanical and physiological parameters, subjective criteria or objective aspects had been solely considered.

Few studies have been conducted to investigate these parameters; however, the mats type, number of participants, data collection method, and performance method have been different in these few studies. The methodology variety has made the obtained results totally different in all of these studies [14]. Based on our knowledge, the effect of anti-fatigue on the variables related to the low back pain due to prolonged standing has not been studied by now. Taking into consideration the significance of the low back pain due to prolonged standing, this experimental study aims to determine the effect of anti-fatigue mats on the low back pain due to prolonged standing on the basis of the VAS and the objective index of bilateral gluteus medius co-activation. Results may be profitable in developing suitable ergonomic approaches to prevent the low back pain due to prolonged standing in individuals who stand fixedly for a long time during a work day.

\section{MATERIAL AND METHODS}

The study was a crossover trial design, and in a doubleblind trial 16 subjects were entered into the trial by a random-number table. Prior to the investigation, volunteers' qualifications for participating in the study had been evaluated. Individuals with the records of the low-back pain, who required medication or 3-month sick absence, the records of hip surgery, and the low-back pain records during the last 12 months were excluded. They were capable of standing more than $4 \mathrm{~h}$. All the subjects participated voluntarily in this study and signed written consent forms approved by the ethical committee of the Urmia University of Medical Sciences.

The study was conducted in two 2-h standing positions including standing on the normal firm surface and on the anti-fatigue floor mat in the ergonomics laboratory. Because of having appropriate thickness and acceptable firmness level [3,14], the ergomat infinity bubble was used as the anti-fatigue floor mat under this study. This product is made of foam polyurethane of $14.5 \mathrm{~mm}$ in thickness. All individuals were positioned in 2 positions in the course of the study. Due to that each day only 2 participants were tested, the break between the tests in position 1 and position 2 for each participant was at least 7 days. In each of the 2 positions, surface areas were covered in order to prevent participants to make judgements subjectively. Data collection methods, and design performance steps in both positions were the same, as described below.

At first, participants were requested to show pain extreme in their low back area by means of the VAS.

Validity and reliability of the VAS were verified by the level of discomfort and low back pain rating in accordance 
with the previous studies [26,27]. In each group of participants, the VAS value obtained prior to standing in both positions was later defined on the basis of 2 criteria. The maximum variation for the VAS scores during 2-h standing was one of the criteria, and the sum of the VAS score variations reported during the 2 positions was the other criterion. The participants were subsequently divided into the pain developer and non-pain developer groups according to the maximum variation for the VAS scores during the 2-h standing. In the pain developer group, the maximum VAS was more than $10 \mathrm{~mm}$, and in the nonpain developer group it was less than $10 \mathrm{~mm}$ [13]. The pain developer group on the normal surface was divided into 2 groups of respondents and non-respondents. Accordingly, if the anti-fatigue floor mat reduced the pain level, they would belong to the respondent group and if it did not - to the non-respondent group.

The electrical activities of gluteus medius muscles were recorded, in accordance with the previous studies, by 1 pair of electrodes $\mathrm{Ag} / \mathrm{AgCl}$ [9-11,13].

The maximum voluntary contraction (MVC) was recorded 3 times in 1-min intervals with 5-s duration on both sides. There was a hand resistance against hip abdaction while the participants were lying on the flanksand.

The raw electromyography signals were collected by using the 8-channel amplified biometreric set model Ltd NP11 $(7 \mathrm{H} \mathrm{z})$, and according to the sampling frequency of $1024 \mathrm{~Hz}$. The biometric set filtered passes of $15-500 \mathrm{~Hz}$ automatically in order to record muscle signals. Although, the exact location of electrodes was defined according to special landmarks, their true locations were controlled by checking output signals when contracting the muscle.

Then, the participants were positioned on a limited and determined place of $64 \times 52 \mathrm{~cm}$ in size. The participants who had worn heel-free sport shoes, were positioned in the naturally anatomical position, and an adjustable table was set in front of them, as such they entered into the experiment protocol in a 2 -h continuous standing position.
Adjustable tables were set $5 \mathrm{~cm}$ below their elbow height. The participants were instructed not to go beyond the delimited area, not to lean their feet on the table feet, and not to incline on the table with a hand. In this study, to simulate the easy professions, 4 various professions such as assembling small parts, classification, mental activities, and standing without doing any activity and social interaction were chosen. These professions were performed randomly for 30 min during 2-h standing, so that the participants were requested to select an action by lot.

Electromyography data was collected from the above mentioned muscles within 15-min successive periods during 2-h standing.

At the end of each period of $15 \mathrm{~min}$, the participants were asked to show the objective pain level by means of the VAS. Finally, 9 pain assessments and 9 muscle electric activity records were developed in 4 random blocks for each position and each participant within the time of $2 \mathrm{~h}$.

At the end, the participants who had stood in both positions were asked which position they preferred.

To analyze and study the EMG signals, a manual program was written in Matlab version R2009a (The Mathworks, Inc., Natick, USA). Since electrodes which were used as software possessed mid-pass filters of $15-500 \mathrm{~Hz}$, no software filters were manually applied to the data. To smooth raw data and data reduction, a time window of $200 \mathrm{~ms}$ was passed for every batch of 30000 data taken for every muscle (left and right) within every $30 \mathrm{~s}(30 \mathrm{~s} \times 1000 \mathrm{~Hz})$ and the root mean square (RMS) was computed for each window. This protocol was applied for 9 times in each test (in 15-min blocks during 2-h standing), and for the triple MVCs data. To normalize the RMS data, according to the equation 1 , all of the RMS data (RMSi) was subtracted from the RMS of the rest data (RMSrest or RMSmin) and divided by the maximum RMS value from the triple MVC tests (RMSMVC or RMSmax) minus the RMS of the rest data (RMSrest or RMSmin). This method was applied to each muscle, and for 9 times in each test. For computing the co-activation 
coefficient index (CCI), which shows simultaneous cocontraction of both muscles quantitatively, the normalized root mean square (NRMS) low(i) and NRMS high(i) were selected from among the NRMS of the 2 muscle groups (right and left gluteus medius) in each test. (In each time 1 of these muscles the normalized root mean square (NRMS low) had the relative minimum value and the other had the relative maximum value (NRMS high)). This process was repeated for 9 steps $(i=1.9)$ that were incorporated into the equation 2 .

So, 9 CCIs were obtained within 25-min intervals for each muscle pair in every position. The mean was computed on the basis of 9 co-activations during 2-h standing as a criterion for comparing the CCI variations in both positions.

The Equi 1 RMS normalizing relation:

$$
\mathrm{NRMS}=\frac{\left(\mathrm{RMS}_{\mathrm{i}}-\mathrm{RMS}_{\min }\right)}{\left(\mathrm{RMS}_{\max }-\mathrm{RMS}_{\min }\right)}
$$

where:

i - data for each session of 9 steps.

The Equi 2 CCI computation relation:

$$
\mathrm{CCI}=\sum\left(\frac{\mathrm{NRMS}_{\text {mini }}}{\mathrm{NRMS}_{\text {maxi }}}\right)\left(\mathrm{NRMS}_{\text {mini }}+\mathrm{NRMS}_{\text {maxi }}\right)
$$

\section{Statistical analysis}

The statistical analysis was performed by means of the SPSS software version 18. In order to test the research hypothesis, the Wilcoxon test, McNemar's test, Mann-Whitney U test, and the repeated measure analysis (RMA) were used. The significance level was $p<0.05$ for all tests.

\section{Findings}

In this study, 1 of the participants was excluded, because his electromyography signals were not appropriate for the analysis purposes.
Visual analogue scale scores during standing

Scores in both positions increased during 2-h standing, and the difference was statistically significant $(\mathrm{p}<0.05)$. As shown in the Figure 1, the VAS score for the position 2 was lower than for the position 1, which was without intervention. By comparison of the mean VAS score changes for the position 1 and 2, the results of the Wilcoxon test along with the significance level of $\alpha=0.05$ revealed that there was a significant difference between the max VAS and the sum of the VAS scores $(p<0.05)$. According to the RMA, there wasn't any statistically significant difference in the mean CCI in bilateral gluteus medius muscles co-activation level in both positions $(\mathrm{p}>0.05)$ as demonstrated in the Figure 2. As compared to the mean gluteus medius muscles co-activation variations in the position 1 and 2, and the Wilcoxon test results in $\alpha=0.05$, it was revealed that unlike the VAS, there was no significant difference between the mean gluteus medius muscles co-activation on both sides in both positions ( $p>0.05$ ).

\section{Low back pain due to standing on the basis of the VAS}

In investigating the low back pain, the participants were divided into the pain developer and the non-pain developer

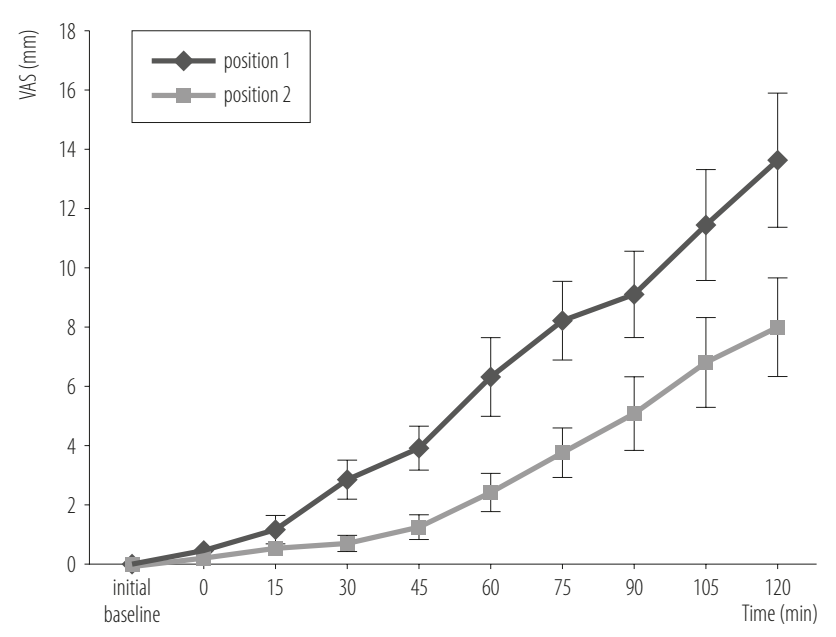

Fig. 1. The process of the visual analogue scale (VAS) score from the baseline during the 2-h standing period in the position 1 and 2 


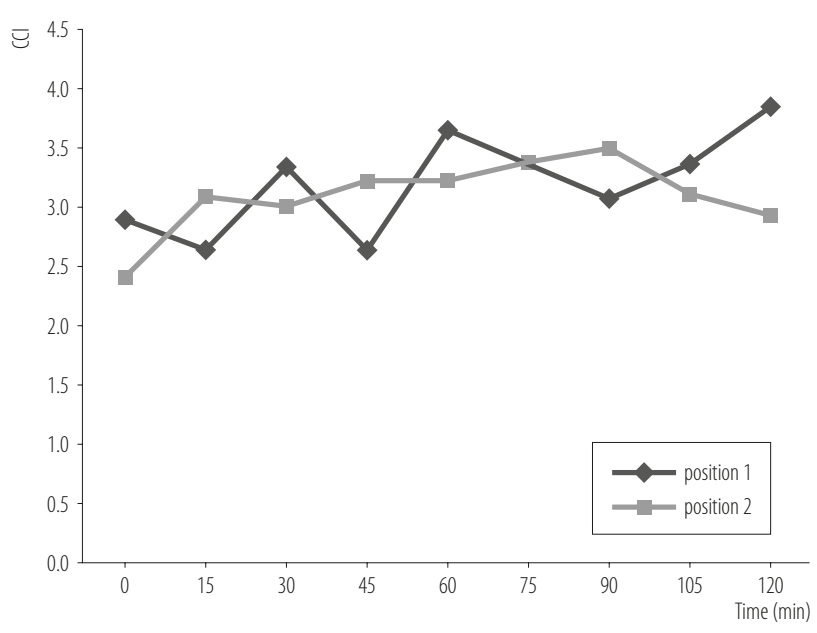

Fig. 2. The process of the bilateral gluteus medius co-contraction index (CCI) during the 2-h standing period in the position 1 and 2

groups according to the VAS data analysis. It was observed that 10 out of 15 participants were in the pain developer group, and the others were in the non-pain developer groups. In the pain developer group, i.e., standing on the anti-fatigue floor mat, there was no significant difference in decreasing the low back pain level as compared to the non-pain developer group ( $p>0.05$ ). The result of the McNemar's test didn't show any significant difference in decreasing the low back pain level due to standing on the anti-fatigue floor mat, when comparing the study groups ( $p>0.05$ ).

General characteristics of the study subjects are presented in the Table 1. According to the Mann-Whitney U test, the participants' characteristics were statistically identical in both groups $(\mathrm{p}<0.05)$. The visual analogue scale variation means for both groups in 2 positions during 2-h standing. The result of the RMA has shown that the VAS mean score is an additional process, and it is influenced by time significantly $(\mathrm{p}<0.05)$. It was lower in the non-pain developer group as compared to the pain developer group (Figures 3 and 4). Similarly, the mean bilateral gluteus medius muscles co-activation was significantly higher in the pain developer group as compared to the non-pain developer group $(\mathrm{p}<0.05)$ (Figures 5 and 6$)$.

By investigating the effect of the anti-fatigue floor mat on the CCI in 10 pain developer participants and 5 non-pain developer participants in the position 1, it was determined that the parameter values changed from $4.35 \pm 0.9$ in the position 1 into $3.82 \pm 0.85$ in the position 2 in the pain developer group, and it changed from $0.92 \pm 0.38$ in the position 1 into $1.63 \pm 0.63$ in the position 2 in the non-pain developer group. According to the Mann-Whitney U test, there wasn't any significant difference between the pain developer and non-pain developer participants in the position $1(\mathrm{p}>0.05)$. Moreover, by studying the effect of the anti-fatigue floor mat on the CCI in 5 respondent participants and 5 non-respondent participants, it was indicated that the $\mathrm{CCI}$ level reduced from $4.31 \pm 1.5$ in the position 1 to $3 \pm 1.04$ in the position 2 in the respondent group, and it was reduced from $4.38 \pm 1.19$ to $4.65 \pm 5.34$ in the non-respondent group in the position 2, although these variations weren't significant $(p>0.05)$.

Table 1. Baseline demographics of the study participants in the pain developer (PD) and non-pain developer (NPD) groups in the position 1 and 2

\begin{tabular}{lccccc}
\hline Standing position & $\begin{array}{c}\text { Group } \\
(\mathrm{N})\end{array}$ & $\begin{array}{c}\text { Age } \\
\text { (years) } \\
(\mathrm{M} \pm \mathrm{SEM})\end{array}$ & $\begin{array}{c}\text { BMI } \\
\left(\mathrm{kg} / \mathrm{m}^{2}\right) \\
(\mathrm{M} \pm \mathrm{SEM})\end{array}$ & $\begin{array}{c}\text { Baseline VAS } \\
(\mathrm{mm})\end{array}$ & $\begin{array}{c}\text { Second baseline VAS } \\
(\text { VAS of min 0) } \\
(\mathrm{M} \pm \mathrm{SEM})\end{array}$ \\
\hline Position 1 & PD 10 & $22 \pm 0.56$ & $21.98 \pm 0.94$ & 0 & $0.4 \pm 0.22$ \\
& NPD 5 & $22.8 \pm 1.36$ & $21.58 \pm 1.08$ & 0 & $0.2 \pm 0.20$ \\
Position 2 & PD 5 & $21.8 \pm 0.58$ & $24.15 \pm 0.99$ & 0 & $0.4 \pm 0.40$ \\
& NPD 10 & $22.5 \pm 0.80$ & $20.69 \pm 0.70$ & 0 & $0.2 \pm 0.13$ \\
\hline
\end{tabular}

VAS - visual analogue scale; M - mean; SEM - standard error of the mean. 


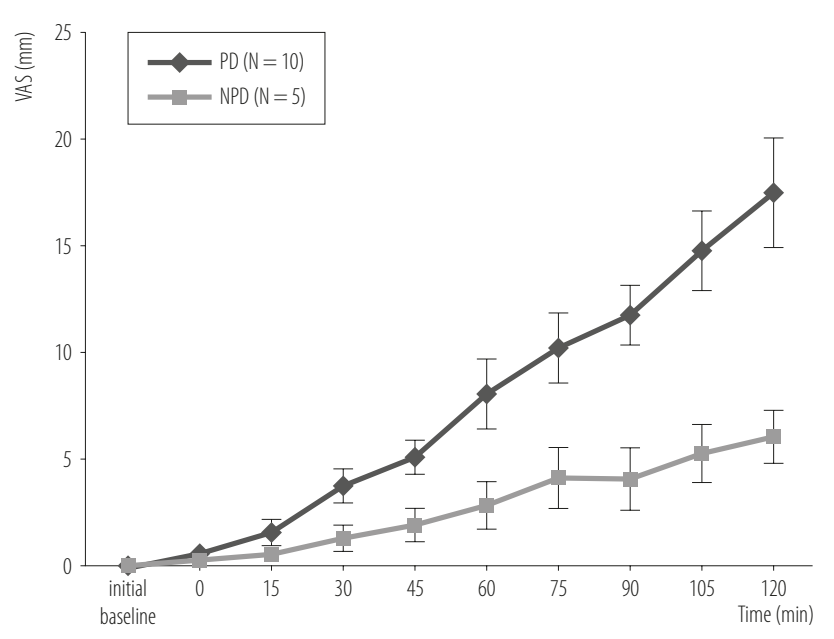

PD - pain developers; NPD - non-pain developers.

Fig. 3. The process of the visual analogue scale (VAS) score from the baseline during the 2-h standing period in the PD and NPD groups in the position 1

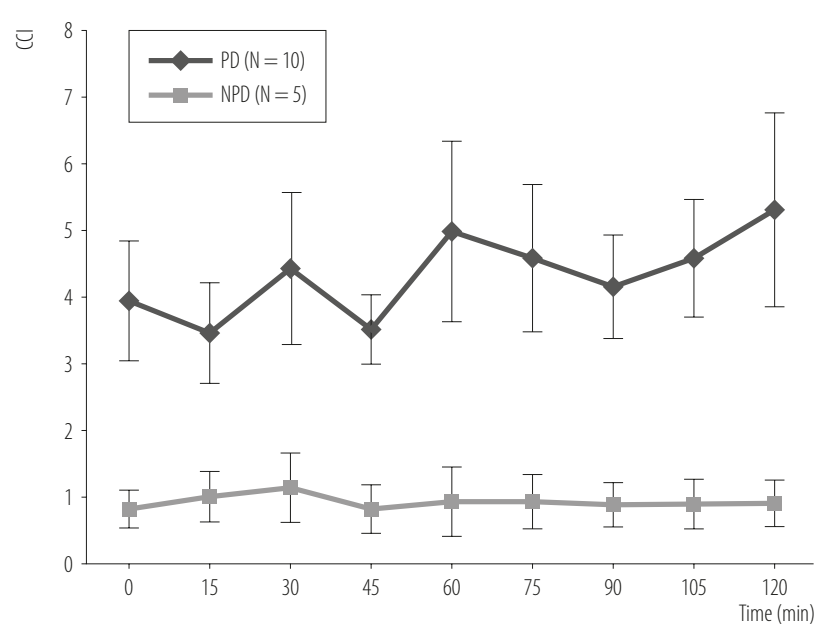

Abbreviations as in the Figure 3.

Fig. 5. The process of the bilateral gluteus medius co-contraction index (CCI) during the 2-h standing period in the PD and NPD groups in the position 1

\section{DISCUSSION}

The results showed that the anti-fatigue floor mat is effective in reducing the low back pain $(p<0.05)$. Based on this study, the anti-fatigue floor mat did not have a significant effect on the objective muscle activity pattern variable related to the low back pain $(\mathrm{p}>0.05)$. Although,

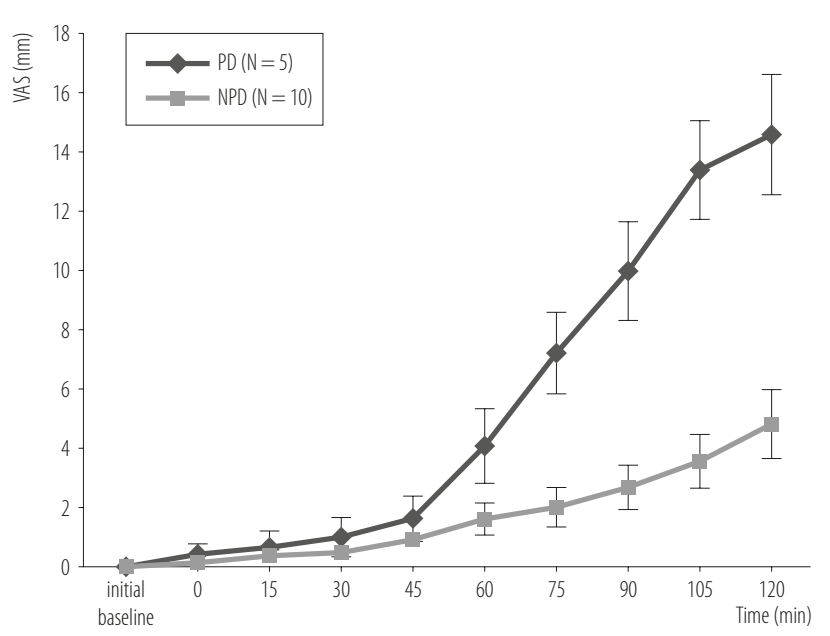

Abbreviations as in Figure 3.

Fig. 4. The process of the visual analogue scale (VAS) score from the baseline during the 2-h standing period in the PD and NPD groups in the position 2

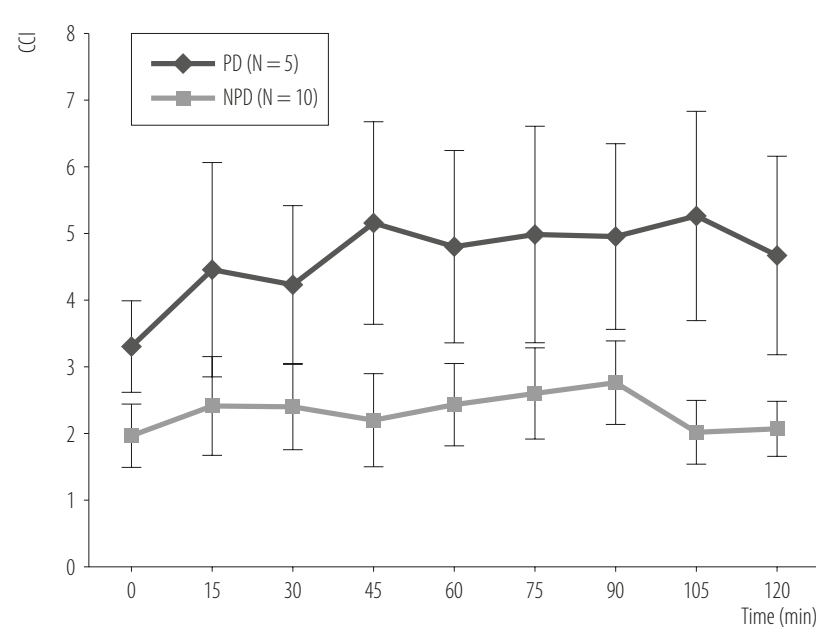

Abbreviations as in the Figure 3.

Fig. 6. The process of the bilateral gluteus medius co-contraction index (CCI) during the 2-h standing period in the PD and NPD groups in the position 2

the anti-fatigue floor mat reduced pain level in 5 members of the pain developer group, it had no significant effect in $\alpha=0.05$ on reducing pain level $(p>0.05)$. Despite increasing the $\mathrm{CCI}$ level for the pain developer group, the anti-fatigue floor mat did not have a significant impact on reducing bilateral gluteus medius muscle co-activation 
in both the pain developer and the non-pain developer groups $(\mathrm{p}>0.05)$.

\section{Low back pain and discomfort during prolonged standing}

In this study, the subjective pain level in low back during 2-h standing was influenced significantly by time $(p<0.05)$. It was increased in low back gradually though pain scale was lower as compared to the position 1 , in the intervention position. This result supported that prolonged standing in static posture is a risky factor in developing low back pain. According to several studies which have been conducted to investigate the relationship between the low back pain and physical factors in various professions such as workers, nurses, surgeries and so on, it has been proven that this factor is functional in developing the low back pain. This study supported the positive impact of the anti-fatigue floor mat on reducing pain, which was in agreement with the studies conducted by Redfern and Chaffin, and Rys and Konz [20,28]. Although, the findings obtained by Konz et al., and Krumwiede et al. revealed that mats have no effect on subjective pain level in the low back pain $[24,29,30]$, this difference could be explained by various standing periods during studies [17].

\section{Bilateral gluteus medius muscle co-activation pattern during standing}

The process of gluteus medius co-activation which is related to the low back pain was not influenced by passing time in both positions $(\mathrm{p}>0.05)$. A significant difference wasn't found in bilateral gluteus medius CCI level between the positions 1 and 2 ( $p>0.05)$. The previous studies proved that, as a determinant factor in developing the low back pain, bilateral gluteus medius muscle co-activation pattern was considered as a dominant pattern for identifying individuals and positioning them in both the pain developer and the non-pain developer groups [9-11,13]. Since participants grouping wasn't done on the basis of susceptiblity to the low back pain intervention in this study, it may lead to decreasing gluteus medius CCI level in the pain developer versus the non-pain developer group [31].

\section{Low back pain due to prolonged standing}

In this study, in the non-intervention position, $67 \%$ of participants suffered from the low back pain and discomfort based on the VAS that was consistent with the others [813]. The results of this study correspond with the studies, in which bilateral gluteus medius co-activation was introduced as a determinant factor in developing the low back pain [9-11,13], and it verified that the co-activation level of the pain developer group for $2 \mathrm{~h}$ was significantly higher as compared to the non-pain developer group. We showed that standing on the anti-fatigue floor mat results in decreasing bilateral gluteus medius muscular co-activation in individuals in the position 1 . However, this increase wasn't significant.

It should be noted that the intervention in the non-pain developer group increased the CCI for gluteus medius. However, there was no increase in the low back pain. No study had been conducted on the effect of the intervention on the low back pain due to prolonged standing using the CCI for gluteus medius. The results obtained from this study correspond with the study performed by Nelson-Wong and Callaghan who investigated the effect of the sloped surface on biomechanics [31]. By objective answers related to standing for $2 \mathrm{~h}$, they found that decreasing the CCI for gluteus medius and objective low back pain in the pain developer group during standing on the sloped surface, it increased in the non-pain developer group without increasing the low back pain [31]. Because of few numbers of the participants, the results of this study can't be generalized to general population. However, there were a few limitations in this study such as the lack of female participants, abnormal shoes of participants, and standing just for $2 \mathrm{~h}$. Despite these limitations, the obtained results provide evidence on the anti-fatigue effect of 
mats in reducing subjective pain level in the low back area. In general, the results support the theory of using mats between foot and firm surface during prolonged standing in order to reduce discomfort in different body areas.

\section{CONCLUSIONS}

This crossover trial study demonstrates that the anti-fatigue floor mats are effective in reducing the low back pain in the visual analogue scale as a subjective measure of the low back pain, but in the objective muscle activity pattern related to the low back pain, they did not have a significant effect on gluteus medius muscle co-activation. Perhaps due to the subjective reduction of the low back pain, the majority of the participants are positive to the application of the anti-fatigue floor mats. The same research should be developed to find out the effect of standing time duration for longer times, for example $4 \mathrm{~h}$. Futhermore, the study can be repeated in the real workplace.

\section{REFERENCES}

1. Shabat S, Gefen T, Nyska M, Folman Y, Gepstein R. The effect of insoles on the incidence and severity of low back pain among workers whose job involves long-distance walking. Eur Spine J. 2005;14(6):546-50, http://dx.doi.org/10.1007/s00586004-0824-z.

2. Nelson-Wong E, Callaghan JP. Changes in muscle activation patterns and subjective low back pain ratings during prolonged standing in response to an exercise intervention. J Electromyogr Kinesiol. 2010;20(6):1125-33, http://dx.doi. org/10.1016/j.jelekin.2010.07.007.

3. King PM. A comparison of the effects of floor mats and shoe in-soles on standing fatigue. Appl Ergon. 2002;33(5):477-84, http://dx.doi.org/10.1016/S0003-6870(02)00027-3.

4. Cook J, Branch TP, Baranowski TJ, Hutton WC. The effect of surgical floor mats in prolonged standing: An EMG study of the lumbar paraspinal and anterior tibialis muscles. J Biomed Eng. 1993;15(3):247-50, http://dx.doi.org/10.1016/0141-5425 (93)90122-F.
5. Kim JY, Stuart-Buttle C, Marras WS. The effects of mats on back and leg fatigue. Appl Ergon. 1994;25(1):29-34, http:// dx.doi.org/10.1016/0003-6870(94)90028-0.

6. Mohseni-Bandpei MA, Fakhri M, Bagheri-Nesami M, Ahmad-Shirvani M, Khalilian AR, Shayesteh-Azar M. Occupational back pain in Iranian nurses: An epidemiological study. Br J Nurs. 2006;15(17):914-7, http://dx.doi.org/10.12968/ bjon.2006.15.17.21904.

7. Mohseni-Bandpei MA, Ahmad-Shirvani M, Golbabaei N, Behtash H, Shahinfar Z, Fernández-de-las-Peñas C. Prevalence and risk factors associated with low back pain in Iranian surgeons. J Manipulative Physiol Ther. 2011;34(6): 362-70, http://dx.doi.org/10.1016/j.jmpt.2011.05.010.

8. Gregory DE, Callaghan JP. Prolonged standing as a precursor for the development of low back discomfort: An investigation of possible mechanisms. Gait Posture. 2008;28(1): 86-92, http://dx.doi.org/10.1016/j.gaitpost.2007.10.005.

9. Nelson-Wong E, Gregory DE, Winter DA, Callaghan JP. Gluteus medius muscle activation patterns as a predictor of low back pain during standing. Clin Biomech (Bristol, Avon). 2008;23(5):545-53, http://dx.doi.org/10.1016/j.clinbiomech.2008.01.002.

10. Marshall PWM, Patel H, Callaghan JP. Gluteus medius strength, endurance, and co-activation in the development of low back pain during prolonged standing. Hum Mov Sci. 2011;30(1):63-73, http://dx.doi.org/10.1016/j.humov.2010.08.017.

11. Nelson-Wong E, Callaghan JP. Is muscle co-activation a predisposing factor for low back pain development during standing? A multifactorial approach for early identification of at-risk individuals. J Electromyogr Kinesiol. 2010;20(2): 256-63, http://dx.doi.org/10.1016/j.jelekin.2009.04.009.

12. Raftry SM, Marshall PWM. Does a "tight" hamstring predict low back pain reporting during prolonged standing? J Electromyogr Kinesiol. 2012;22(3):407-11, http://dx.doi. org/10.1016/j.jelekin.2012.02.008.

13. Nelson-Wong E, Callaghan JP. Repeatability of clinical, biomechanical, and motor control profiles in people 
with and without standing-induced low back pain. Rehabil Res Pract. 2010;2010:289278, http://dx.doi.org/10.1155/ 2010/289278.

14. Orlando AR, King PM. Relationship of demographic variables on perception of fatigue and discomfort following prolonged standing under various flooring conditions. J Occup Rehabil. 2004;14(1):63-76, http://dx.doi.org/10.1023/ B:JOOR.0000015011.39875.75.

15. Almeida JS, Filho GC, Pastre CM, Padovani CR, Martins RADM. Comparison of plantar pressure and musculoskeletal symptoms with the use of custom and prefabricated insoles in the work environment. Rev Bras Fisioter. 2009;13(6):542-8.

16. Basford JR, Smith MA. Shoe insoles in the workplace. Orthopedics. 1988;11(2):285-8.

17. Cham R, Redfern MS. Effect of flooring on standing comfort and fatigue. Hum Factors. 2001;43(3):381-91, http:// dx.doi.org/10.1518/001872001775898205.

18. Hansen L, Winkel J, Jørgensen K. Significance of mat and shoe softness during prolonged work in upright position: Based on measurements of low back muscle EMG, foot volume changes, discomfort and ground force reactions. Appl Ergon. 1998;29(3):217-24, http://dx.doi.org/10.1016/ S0003-6870(97)00062-8.

19. Kelaher D, Mirka GA, Dudziak KQ. Effects of semi-rigid arch-support orthotics: An investigation with potential ergonomic implications. Appl Ergon. 2000;31(5):515-22, http:// dx.doi.org/10.1016/S0003-6870(00)00018-1.

20. Redfern MS, Chaffin DB. Influence of flooring on standing fatigue. Hum Factors. 1995;37(3):570-81.

21. Zander JE, King PM, Ezenwa BN. Influence of flooring conditions on lower leg volume following prolonged standing. Int J Ind Ergon. 2004;34(4):279-88, http://dx.doi. org/10.1016/j.ergon.2004.04.014.
22. Zhang L, Drury CG, Woolley SM. Constrained standing: Evaluating the foot/floor interface. Ergonomics. 1991;34(2): 175-92, http://dx.doi.org/10.1080/00140139108967305.

23. Madeleine P, Voigt M, Arendt-Nielsen L. Subjective, physiological and biomechanical responses to prolonged manual work performed standing on hard and soft surfaces. Eur J Appl Physiol and Occup Physiol. 1998;77(1-2):1-9, http://dx.doi.org/10.1007/s004210050292.

24. Konz S, Bandla V, Rys M, Sambasivan J. Standing on concrete vs. floor mats. London: Taylor \& Francis; 1990. p. 991-8.

25. Rys M, Konz S. Standing. Ergonomics. 1994;37(4):677-87, http://dx.doi.org/10.1080/00140139408963682.

26. Revill SI, Robinson JO, Rosen M, Hogg MI. The reliability of a linear analogue for evaluating pain. Anaesthesia. 1976;31(9):1191-8, http://dx.doi.org/10.1111/j.1365-2044. 1976.tb11971.x.

27. Summers S. Evidence-based practice part 2: Reliability and validity of selected acute pain instruments. J Perianesth Nurs. 2001;16(1):35-40, http://dx.doi.org/10.1016/S10899472(01)19774-1, http://dx.doi.org/10.1053/jpan.2001.20657.

28. Rys M, Konz S. An evaluation of floor surface. In: Proceedings of Human Factors Society 33rd Annual Meeting. Human Factors Society; 1989. p. 517-520.

29. Krumwiede D, Konz S, Hinnen P. Floor mat comfort. In: Kumar S, editor. Advances of occupational ergonomics and safety. Amsterdam: IOS Press; 1998. p. 159-62.

30. Rys M, Konz S. Standing work: Carpet vs. concrete. In: Proceedings of Human Factors Society 32nd Annual Meeting. Human Factors Society; 1988. p. 522-26.

31. Nelson-Wong E, Callaghan JP. The impact of a sloped surface on low back pain during prolonged standing work: A biomechanical analysis. Appl Ergon. 2010;41(6):787-95, http://dx.doi.org/10.1016/j.apergo.2010.01.005.

This work is available in Open Access model and licensed under a Creative Commons Attribution-NonCommercial 3.0 Poland License - http://creativecommons.org/ licenses/by-nc/3.0/pl/deed.en. 